CERN-TH/2000-083

hep-th/0003198

\title{
On Supersymmetry Breaking in String Theory and its Realization in Brane Worlds
}

\author{
P. Mayr \\ CERN Theory Division \\ CH-1211 Geneva 23 \\ Switzerland
}

\begin{abstract}
We use string duality to describe instanton induced spontaneous supersymmetry breaking in string compactifications with additional background fields. Dynamical supersymmetry breaking by space-time instantons in the heterotic string theory is mapped to a tree level breaking in the type II string which can be explicitly calculated by geometric methods. The point particle limit describes the non-perturbative scalar potential of a SYM theory localized on a hypersurface of space-time. The $\mathcal{N}=0$ vacuum displays condensation of magnetic monopoles and confinement. The supersymmetry breaking scale is determined by $M_{s t r}$, which can be in the $\mathrm{TeV}$ range, and the geometry transverse to the gauge theory.
\end{abstract}

March 2000 


\section{Introduction and summary}

The remarkable success in the understanding of non-perturbative properties of string theories triggered by string dualities can not conceal the fact that their impact on the formulation of theories with $N<2$ supersymmetry and dynamical supersymmetry breaking has been much less effective. In fact the step to $N=1$ supersymmetry may be already a vital one for the description of the non-supersymmetric world as it is known that dynamical supersymmetry breaking may appear in these theories as a consequence of strong gauge interactions [1]. It is interesting that supersymmetry breaking in the string theory might be so closely related to its low energy sector. In fact in the realization of confinement as result of monopole condensation [2] [3], anomaly considerations imply a non-zero gaugino condensate [4 which breaks supersymmetry in the theory coupled to gravity [1]. In this sense the lack of supersymmetry observed in our world could be linked in an intriguing way to the existence of confining gauge theories.

One of the most useful $N=2$ dualities has been the one between type II and heterotic strings [5] [6]. As the heterotic coupling maps to a geometric modulus in the type II theory, non-perturbative effects of the heterotic theory are related to tree level of the type II string. In particular space-time instanton effects map to geometric instantons in the type II theory which can be calculated by mirror symmetry. More fundamentally the instantons in the type II theory can be viewed as honest space-time instantons in the RR-sector, which has the special property to not depend on the type II string coupling and thus is described by string tree level.

This opens the fascinating possibility that after a supersymmetry breakdown to $\mathcal{N}=1$, dynamical supersymmetry breaking to $\mathcal{N}=0$ in the RR-sector of the type II theory is calculable as the non-perturbative effects are still governed by a geometric coupling constant and not the string coupling. In particular condensation of fermions will appear at string tree level. This non-supersymmetric type II theory will be dual to a $\mathcal{N}=1$ supersymmetric heterotic theory where supersymmetry is broken only in the non-perturbative sector of the string theory. Such a picture was advocated in [7] using the special constructions of [8] to obtain $\mathcal{N}=1$ dual pairs from $\mathcal{N}=$ 2 ones by freely acting orbifoldst. Based on qualitative arguments on the general properties of the orbifolding procedure it was argued that indeed gaugino condensation

1 The relation between spontaneous supersymmetry breaking in string theory and freely acting orbifolds with various supersymmetries has been studied in refs. [9]. 
in the heterotic theory should map to a tree level supersymmetry breaking in the type II theory which, remarkably enough, was related to monopole condensation and the associated relalization of confinement. Unfortunately the orbifold duals of [7] are not accessible to instanton calculations by mirror symmetry and thus the study of string duals with supersymmetry breaking has been restricted to a rather qualitative level, far from the success in the $\mathcal{N}=2$ supersymmetric case. To provide a calculable framework of string duals with dynamical supersymmetry breaking is one of the goals of this paper.

In a seemingly unrelated development in the supergravity part of the world, the no-go theorem [10] on partial local supersymmetry breaking $N=2 \rightarrow N=1$ was deprived its validity [11]. The argument is that for a special class of supergravity theories, the tensor calculus of ref. [12], which was the basis for the derivation of the no-go theorem, is inappropriate. In these special cases, the no-go theorem does not apply and spontaneous partial supersymmetry breaking has indeed be shown to be possible [11]. In string theory compactifications, scalar potentials that may trigger spontaneous supersymmetry breaking can be induced by RR background fields in the type II string [13] or magnetic backgrounds in the dual heterotic theory [14]. However, as we will argue in this paper, partial breaking fails to exist, although in an interesting way. The reason is that even though the conditions of partial supersymmetry breaking may be satisfied in the classical string effective theory, there will always be instanton effects in compactifications with finite volume, that restore the foundations of the no-go theorem. We will also argue that the effect of these instantons is to break supersymmetry completely, rather then to restore $\mathcal{N}=2$.

An interesting picture begins to emerge by observing that the classical string effective theories associated to $N=2$ type II/heterotic duals fall precisely into the class of supergravities that satisfy the necessary conditions for partial supersymmetry breaking. Would it not be for the instanton effects, we would get $\mathcal{N}=1$ supersymmetric dual pairs in this way. Turning to the instanton corrections one observes that they represent non-perturbative space-time effects in the heterotic string and tree level geometric instantons in the type II theory. In other words, the instanton effects that restore the no-go theorem embody precisely the sought for description of non-perturbative dynamical supersymmetry breaking. Using mirror symmetry in the tree level type II theory to determine the geometric instantons we are thus in the remarkable situation to be able to systematically calculate the instanton effects of a phenomenologically relevant non-perturbative supersymmetry breaking. 
Interestingly, and in full agreement with the observations made in the free orbifold models of [7], the supersymmetry breaking is intimately related to strong gauge interactions in a SYM theory embedded into the string theory. In fact this theory is of "the world on a brane" type, with the gauge fields localized on a hypersurface of ten-dimensional space-time. These theories have been very successfully studied in the $\mathcal{N}=2$ context in terms of curved brane geometries in string theory, either in the geometric engineering of type II strings on singularities of Calabi-Yau 3-folds [15] 16] 17] or, for simple enough gauge group $G$, in terms of the T-dual type IIA 5-brane [15]. The latter realization can also be reached starting from field theories living on the world-volume of "flat branes" which are bent by quantum corrections in a way determined by the non-perturbative duality to M-theory [18]. Both, the "flat" and the curved brane constructions, give a string theory realization of the intensively discussed extra large dimension scenarios [19]. In particular the "flat branes" have become a most popular tool in qualitative phenomenological studies due to their conceptional simplicity as compared to the geometric approach.

So finally, taking the point particle limit of the eventually non-supersymmetric dual pair, leads us right to an extension of geometric engineering to more realistic SYM theories on the brane, with broken supersymmetry and patterns of confinement

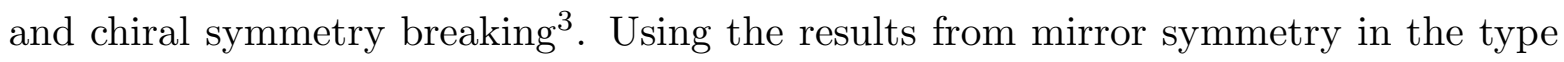
II theory allows to derive the explicit non-perturbative scalar potential in the SYM theory on the brane.

It is worth noting that the description of supersymmetry breaking in the SYM theory on the brane is quite relevant for the large dimension scenarios one of whose primary motivations has been the hierarchy problem. A serious attempt to explain the apparent weakness of gravity should be based on a framework of quantum gravity such as string theory, and not just field theories on "flat branes". Note that the resurrection of the no-go theorem implies that the complete breakdown to $\mathcal{N}=0$ (rather than $\mathcal{N}=1$ ) is inevitable when the SYM is coupled to string theory. It would be quite interesting to develop the understanding of the field theories based "flat branes" with $\mathcal{N}=1$ supersymmetry [21] to a level where the supersymmetry

2 A different approach uses F-theory compactifications [20].

3 In the following we will use the definiton of a brane in the general sense of specifying a hypersurface on which the gauge fields localize. This notion is independent of the specific string theory construction in terms of dual D-branes, M-branes or geometrically engineered theories at a singularity. 
breakdown can be seen, at least qualitatively. The reason that the flat branes miss this effect is apparently the lack of a satisfying description of the coupling of the world volume theory to gravity and string theory.

Note that we argued that the restoration of the no-go theorem relies on stringy instantons on a finite volume manifold. This does not exclude partial supersymmetry breaking in diverse non-compact limits. In particular, taking all, transverse as well as tangential dimensions of infinite volume gives a pure field theory decoupled from strings and gravity. It should be clear that neither there is a no-go theorem in pure field theory nor do we claim so 1 . In fact, while we completed this work, an interesting paper appeared [23], which also describes supersymmetry breaking by RR fluxes in the context of geometric engineering. In ref. 23] the limit of infinite string mass $M_{s t r}$ is taken which results in a pure field theory decoupled from string theory where partial breaking is possible. Moreover this reference contains an elegant derivation of how the relevant $\mathcal{N}=1$ superpotentials follow from soliton masses in the presence of RRpotentials.

This paper is organized in two parts. In the first part we consider the issue of partial supersymmetry breaking in the string effective supergravity. In section 2 we argue that for string compactifications on manifolds with finite volume, any classical partial breaking to $\mathcal{N}=1$ is followed by a further breakdown to $\mathcal{N}=0$ by instanton effects. We resurrect the general no-go theorem on partial breaking for string effective theories. In section 3 we relate these results to the class of string effective theories which reduce to ordinary Super-Yang-Mills theories in the point particle limit. The instantons that are relevant for the supersymmetry breakdown turn out to be nonperturbative in the field theory coupling. In section 4 we describe in more detail the mechanism of supersymmetry breaking and the calculation of the non-perturbative gravitino masses by mirror symmetry. Moreover we observe a general link between the breakdown of supersymmetry and that of conformal invariance.

In the second part, which starts with section 5 and can be read almost independently, we focus further on the pattern of supersymmetry breaking in the point particle limit, namely in the SYM on the brane embedded in the string theory. The general non-perturbative scalar potential contains apart from the $\mathcal{N}=1$ adjoint mass term extra soft breaking terms that arise from the coupling to the string sector. We use special geometry to proof that these terms are mandatory and there is no partial

\footnotetext{
4 E.g., gaugino condensation does not break supersymmetry in field theory [22].
} 
breaking to $\mathcal{N}=1$ in the field theory coupled to string theory. The scale of supersymmetry breaking terms is determined by the string scale $M_{s t r}$, which is argued to be favorably in the $\mathrm{TeV}$ range, multiplied by a factor $\sim b_{1} \cdot V_{T V}^{-1}$, where $b_{1}$ is the one-loop beta function coefficient of the SYM theory and $V_{T V}$ the volume of the dimensions transverse to the gauge theory. Details on the supergravity calculations are relegated to the appendices.

\section{Partial supersymmetry breaking in $\mathcal{N}=2$ supergravity (or "Two into one still won't go")}

Before focusing on the string theory embeddings of SYM theories let us ask in general what patterns of supersymmetry breaking may appear in the $\mathcal{N}=2$ string effective theory. As far as partial local supersymmetry breaking by a Super-Higgs effect is concerned, there used to be a very strong result, the "Two into one won't go" theorem of [10]. It states that a zero eigenvalue of the gravitino mass matrix implies that the second one is zero, too.

However, the no-go theorem is based on the existence of a holomorphic prepotential $\mathcal{F}\left(z_{i}\right)$ that defines the effective $\mathcal{N}=2$ supergravity action of the $n_{V}$ vector multiplets. At that time this was indeed the only known description of the $\mathcal{N}=2$ supergravity theory [12]. Since then, an alternative definition has been formulated which is not based on a prepotential [24]. It thus appeared that the absence of a prepotential might possibly allow to evade the no-go theorem and indeed it was shown in [1] that partial supersymmetry breaking is possible in this special situation. The globally supersymmetric version of this partial breaking has been described independently in 25.

The necessary condition for the absence of a prepotential and thus partial supersymmetry breaking is the following one. The framework of [24] starts from a section $\Pi=\left(X^{\Sigma}, F_{\Sigma}\right)^{T}$ of a $S p\left(2 n_{V}+2, \mathbf{Z}\right)$ bundle over $M_{V}$. It is invariant under symplectic transformations acting on $\Pi$ :

$$
\Pi \rightarrow M \Pi, \quad M \in S p(2 n+2, \mathbf{Z}) .
$$

The components $X^{\Sigma}, F_{\Sigma}$ of the section $\Pi$, also called periods, depend on the $n_{V}$ scalar components $z_{i}$ of the vector multiplets which parametrize an $n_{V}$ dimensional special Kähler manifold $\mathcal{M}_{V}$. In a generic situation the upper components $X^{\Sigma}$ can be thought of as homogeneous coordinates on $\mathcal{M}_{V}$, while the lower half $F_{\Sigma}$ of $\Pi$ is related to a 
prepotential $\mathcal{F}\left(X^{\Lambda}\right)$ of homogeneous degree two by $F_{\Sigma}=\partial \mathcal{F} / \partial X^{\Sigma}$. The transition to the generic inhomogeneous variables $z_{i}$ on $M_{V}$ is described by the matrix

$$
A^{\Sigma i}=\frac{\partial X^{\Sigma}}{\partial z_{i}}
$$

For a special form of the prepotential $\mathcal{F}$ and a special choice of the section $\Pi$, the matrix $A^{\Sigma i}$ may be degenerate. Then the $X^{\Sigma}$ can not serve as homogeneous coordinates on $\mathcal{M}_{V}$ and no prepotential $\mathcal{F}\left(X^{\Lambda}\right)$ exists. Note that this statement is not invariant under $S p(2 n+2, \mathbf{Z})$ transformations and by choosing a different section $\tilde{\Pi}=M \Pi$ one may always transform to homogeneous coordinates $\tilde{X}^{\Sigma}$ in which a prepotential $\tilde{\mathcal{F}}\left(\tilde{X}^{\Sigma}\right)$ exists.

A string effective supergravity related to the geometric type II compactification on a Calabi-Yau manifold $M$ is classically described by a cubic prepotential

$$
\mathcal{F}=\frac{1}{3 !} C_{i j k} X^{i} X^{j} X^{k} / X^{0}=\left(X^{0}\right)^{2} \frac{1}{3 !} C_{i j k} t_{i} t_{j} t_{k}
$$

where $t_{i}=X^{i} / X^{0}$ are so-called special coordinates that parametrize volumes of homology 2-cycles in $M$. Depending on the intersection matrix $C_{i j k}$ of $M$ it may be possible to choose a section $\Pi$ where the $X^{\Sigma}$ are dependent, the matrix $A^{\Sigma i}$ is degenerate and partial breaking to $\mathcal{N}=1$ supersymmetry is possible 5 .

However in a finite volume there are stringy instanton corrections to the classical prepotential (2.3) that change this picture. The exact instanton corrected prepotential can be determined [26] using mirror symmetry6. Specifically, the components of $\Pi$ are identified under mirror symmetry with the period integrals of the holomorphic 3-form $\Omega^{3,0}$ on the mirror manifold $W$ of $M$ :

$$
X^{\Sigma}=\int_{A^{\Sigma}} \Omega^{(3,0)}(W), \quad F_{\Sigma}=\int_{B_{\Sigma}} \Omega^{(3,0)}(W) .
$$

Here $\left\{A^{\Sigma}\right\}$ denotes an integral basis of homology 3-cycles and $\left\{B_{\Sigma}\right\}$ their duals. The period integrals $F_{\Sigma}$ have the large $t_{i}$ expansion

$$
F_{\Sigma}=\frac{1}{2 !} C_{i j k} t_{j} t_{k}+f\left(t_{n}, q_{n}\right)
$$

5 We will argue that this happens for string theory embeddings of SYM theories in the next section.

${ }^{6}$ For an overview and references, see [27]. For a recent proof in the supersymmetric sigma model, see [28]. 
where $q^{n}=\exp \left(2 \pi i t_{n}\right)$ are the instanton corrections suppressed by the exponential of the Kähler volumes of 2 -spheres.

We want to argue now that even in a situation where the geometrical data $C_{i j k}$ allow for a partial supersymmetry breaking to $\mathcal{N}=1$, the stringy instanton corrections always lead to a complete breakdown of supersymmetry. To do so we have to assure

that after the inclusion of the instanton corrections, the upper part $\hat{X}^{\Sigma}$ of any section $\hat{\Pi}$ gives good homogeneous coordinates on $\mathcal{M}_{V}$. By the relation (2.4) this is the same as asking whether for any choice of a basis of $A$-cycles in (2.4), the period integrals represent locally good homogeneous coordinates on $\mathcal{M}_{V}$. Luckily this type of question is a prominent one in algebraic geometry and is part of the so-called infinitesimal Torelli problem. In the present context it has been shown in ref. [29] that the period integrals indeed have the required property.

In conclusion, although partial supersymmetry breaking may appear to be possible in the classical string theory, there will be always stringy instanton corrections that lead to a complete breakdown of supersymmetry and the mechanism of refs. 11] can not be realized in the string effective theory.

\section{Localized SYM theories and their string theory embedding}

Form the point of physics, the situation that the classical section $\Pi$ allows for partial supersymmetry breaking while the instanton corrected one does not is quite a remarkable one. Specifically the supersymmetry breaking scale will depend nonperturbatively on the Kähler moduli. We will argue now that this is the case in the class of string effective theories realizing SYM theories localized on a six-dimensional hypersurface of space-time. As we will discuss in the following, the relevant modulus is identical to the field theory coupling constant and the supersymmetry breakdown is thus interpreted as a non-perturbative breaking by space-time instantons. 


\subsection{Geometry and couplings}

The geometric engineering approach [15][16] [17] starts from a type II string compactified on an almost singular Calabi-Yau manifold $X$ Z. It is useful to think about this compactification as a two step process of a compactification to six dimensions on a singular two complex-dimensional manifold $Y$ followed by a further compactification on the base sphere $B$ to four dimensions. At the singular point $p$ of $Y$ there are $r=\operatorname{rk} G$ small 2-spheres $C_{i}$ with an intersection matrix equal to the negative of the Cartan matrix $C_{i j}^{G}$. D2-branes wrapped on the 2 -spheres $C_{i}$ represent $\mathcal{N}=2$ vector multiplets in six dimensions with a gauge kinetic term (in a tree level approximation)

$$
\mathcal{L}_{k i n}=\frac{1}{4} C_{i j} F^{(i) \mu \nu} F_{\mu \nu}^{(j)}
$$

In particular the effective action is independent of the directions transverse to the singularity, as is expected from the fact that the gauge fields are localized on the six-dimensional hypersurface specified by $p \subset Y$. This gives a natural string theory realization of the (possibly large) extra dimension scenario of [19].

Upon compactification on the sphere $B$ one obtains an $\mathcal{N}=2$ supersymmetric QFT with gauge group $G$ in four dimensions. Dimensional reduction implies that the four-dimensional tree level gauge coupling is given by the (complexified) Kähler volume $V_{B}$ of $\mathrm{B}$ :

$$
\frac{\theta_{F T}}{2 \pi}+\frac{4 \pi i}{g_{F T}^{2}}=\int_{B} B_{N S}+i V_{B} \equiv s .
$$

The real part of $s$ describes the integral of the Neveu-Schwarz $B$-field on the base 2-sphere $B$.

At this point it seems useful to fix the notation for the coupling constants. In the following we will refer to the field theory coupling constant $s$ as "the coupling constant" and use "perturbative" with respect to the the field theory which is designed to describe our world. The string theory embedding with $s$ identical to the string coupling constant is a heterotic string on $\mathrm{K} 3 \times T^{2}$. It is dual [5] [6] to the geometric type II compactification where $s$ is the geometric volume (3.2) and not the string coupling. This is the usual story used in geometric engineering: we can calculate non-perturbative phenomena of the effective field theory by using the geometric type II picture. In the present paper we extend this correspondence to also calculate nonperturbative quantities of the string effective supergravity theory to which the SYM theory is coupled.

\footnotetext{
7 For a basic introduction and further references, see [30].
} 


\subsection{The string effective supergravity}

The four-dimensional tree level coupling (3.2) fixes the classical piece of the prepotential to be

$$
\mathcal{F}=-s C_{i j} t_{i} t_{j}+\ldots,
$$

where the dots denote $\alpha^{\prime}$ and finite coupling corrections. It is easy to see that the string effective supergravity obtained from geometric engineering has precisely the property to allow for a partial supersymmetry breaking. In the inhomogenous coordinates $t_{i}, s$, the standard section $\Pi=\left(X^{\Sigma}, \partial \mathcal{F} / \partial X^{\Sigma}\right)$ is

$$
X^{\Sigma}=\left(1, s, t_{i}\right), \quad F_{\Sigma}=\left(2 \mathcal{F}-s \mathcal{F}_{s}-\sum_{k} t_{k} \mathcal{F}_{k}, \mathcal{F}_{s}, \mathcal{F}_{i}\right),
$$

where subscripts denote differentiation. From the prepotential (3.3) one obtains after a simple symplectic transformation $\left(s, \mathcal{F}_{s}\right) \rightarrow\left(\mathcal{F}_{s},-s\right)$ a section $\tilde{\Pi}$ with

$$
\tilde{X}^{\Sigma}=\left(1, \mathcal{F}_{s}, t_{i}\right)^{T}, \quad \tilde{F}_{\Sigma}=\left(2 \mathcal{F}-s \mathcal{F}_{s}-\sum_{k} t_{k} \mathcal{F}_{t_{k}},-s, \mathcal{F}_{i}\right)^{T},
$$

In particular

$$
\mathcal{F}_{s}=\partial \mathcal{F} / \partial s=-C_{k l} t_{k} t_{l} .
$$

Note that the $\tilde{X}^{\Sigma}$ do not depend on $s$ and thus the necessary condition for partial supersymmetry breaking is satisfied.

However, as we argued on general grounds in section 2, instanton corrections will change this picture. In the present situation the relevant instantons have an action

$\sim e^{2 \pi i s}$ which is characteristic of space-time instantons as interpreted in the field theory. It is one of the most powerful aspects of the geometrically engineered quantum field theories (GEQFT's) that one may determine these non-perturbative corrections from the dual, geometric type II instantons by mirror symmetry. We will use this information in the next section to study the pattern of supersymmetry breaking in the exact string effective supergravity theory.

\section{Non-perturbative supersymmetry breaking in the string effective theory}

Apart from the vector multiplets, the general $\mathcal{N}=2$ supergravity can be coupled to $\mathcal{N}=2$ matter hypermultiplets. These couplings may generate a non-trivial scalar potential which determines the vacuum structure. We review a few facts and definitions in appendix A. In string theory these couplings may arise as the consequence of background fields such as RR fields in type II strings [13] and magnetic backgrounds in the heterotic string [14]. In the following we study the contribution of instanton effects in the presence of such backgrounds. 


\subsection{The $S U(2)$ GEQFT on a compact manifold}

Let us first consider a global type II compactification associated to the $S U(2)$ GEQFT as an explicit example for the supersymmetry breaking by instantons on a compact manifold. It will be rather obvious how to generalize to the generic situation subsequently.

The prepotential of the string effective supergravity is of the form

$$
\mathcal{F}(s, t)=-s\left(t^{2}+1\right)-a t^{3}+b t+c+f(s, t), \quad a, b, i c \in \mathbf{R} .
$$

The modulus $t$ is related to the single 2-sphere of the singularity supporting the $S U(2)$ theory. In the tree level approximation $s \rightarrow \infty$, eq.(4.1) is the same as (3.3) for $G=S U(2)$ up to a shift of $t$ by a constant. This shift is an effect of the global geometry. It has no further consequences except for the translation of the origin of the $S U(2)$ theory to $t=i$. The function $f$ depends only on the exponentials $\left(q_{s}, q_{t}\right)=\left(e^{2 \pi i s}, e^{2 \pi i t}\right)$. The $s$-independent piece of $\mathcal{F}(s, t)$ describes one-loop effects in the heterotic string theory whereas a term $\sim q_{s}^{k}$ corresponds to an $k$ instanton effect.

The presence of background fields induces charges of a hypermultiplet w.r.t. to a gauge symmetry gauged by the vector multiplets. Let us first ask what kind of gauge charges will be relevant. In the Super-Higgs effect, one gravitino becomes part of a massive super multiplet with spin content $(3 / 2,1,1,1 / 2)$ [12]. It is natural to identify these two spin one vectors with the ones in the universal sector of the graviphoton and the dilaton $s, \Sigma=0,1$. Note that this choice is universal and is valid for any gauge group $G$. In addition there may be an ordinary Higgs effect in the magnetic field theory $U(1)$ factors related to the condensation of monopoles.

To proceed we perform a symplectic transformation on the standard section (3.4) and obtain a period vector $\tilde{\Pi}$ of the form

$$
\tilde{X}^{\Sigma}=\left(\begin{array}{c}
-\frac{1}{2}\left(t^{2}-1\right)+\frac{1}{2} f_{s} \\
-t \\
-\frac{1}{2}\left(t^{2}+1\right)+\frac{1}{2} f_{s}
\end{array}\right), \tilde{F}_{\Sigma}=\left(\begin{array}{c}
-t^{2} s+a t^{3}-b t+s-2 c-2 f+t f_{t}+s f_{s} \\
-2 t s+3 a t^{2}+b+f_{t} \\
t^{2} s-a t^{3}+b t+s+2 c+2 f-t f_{t}-s f_{s}
\end{array}\right) .
$$

This basis is the equivalent of (3.5) in the global compactification. Note that the $s$ dependend piece of $\tilde{X}^{\Sigma}$ is entirely due to the space-time instantons correction $f_{s}=$

$\partial f / \partial s \sim q^{s}=e^{2 \pi i s}+\ldots$. Therefore in the perturbative, tree-level plus one-loop corrected supergravity, no prepotential exists and partial supersymmetry breaking appears to be possible. 


\subsection{Non-perturbative gravitino masses}

However, as asserted already, including the space-time instantons, the $X^{\Sigma}$ should be always good homogeneous coordinates and the arguments of the no-go theorem [10] apply. To be concrete, let us consider a breaking to $\mathcal{N}=1$ near the origin $t=i$ of the field theory Coulomb branch. The gravitino mass matrix is proportional to the supersymmetry variation $S_{A B}$ :

$$
i S_{A B}=-\frac{i}{2} e^{K / 2}\left(\begin{array}{cc}
P_{0}^{1}-i P_{1}^{1}-i P_{0}^{1}-P_{1}^{2} & -P_{0}^{3}+i P_{1}^{3} \\
-P_{0}^{3}+i P_{1}^{3} & -P_{0}^{1}+i P_{1}^{1}-i P_{0}^{2}-P_{1}^{2}
\end{array}\right)
$$

A representative chocie for a breaking to $\mathcal{N}=1$ with a zero single eigenvalue of the matrix $S$ is

$$
P_{0}^{1}=P_{1}^{2}=m, \quad P_{\Sigma}^{x}=0 \text { for } \Sigma=0,1, \text { else. }
$$

With this choice the mass matrix for generic moduli is

$$
i S_{A B}=\frac{i m e^{K / 2}}{4}\left(\begin{array}{cc}
(t-i)^{2}-f_{s} & 0 \\
0 & -(t+i)^{2}+f_{s}
\end{array}\right)
$$

For $q_{s} \rightarrow 0$ there is a $\mathcal{N}=1$ vacuum at $t=i$. For $q_{s} \neq 0$ the instantons lift the zero eigenvalue. Since the no-go theorem applies, there must be either $\mathcal{N}=2$ or $\mathcal{N}=0$ supersymmetry. An $\mathcal{N}=2$ vacuum requires $t=0, f_{s}=-1$. Even if such a point in the moduli space would exist, it would not be connected to the large $s$ vacuum. Thus the instantons break further $\mathcal{N}=1 \rightarrow \mathcal{N}=0$ at a scale $\Lambda \sim e^{-8 \pi^{2} / g^{2}}$.

The exciting fact about this instanton generated supersymmetry breaking is that the non-perturbative effects are determined by mirror symmetry in the geometric type II theory. Since the non-perturbative gravitino masses arise from the coupling of the GEQFT to the string and gravity sector, the precise instanton series depends on the individual compactification manifold M. As an example let us consider the mirror of the Calabi-Yau manifold 8 defined by the polynomial

$$
p=x_{1}^{12}+x_{2}^{12}+x_{3}^{6}+x_{4}^{6}+x_{5}^{2}-12 \mu x_{1} x_{2} x_{3} x_{4} x_{5}-2 \phi x_{1}^{6} x_{2}^{6} .
$$

Here the $x_{i}$ are coordinates of a weighted projective space in which $M$ is embedded as the hypersurface $p=0$ and $(\mu, \phi)$ are coordinates on $\mathcal{M}_{V}(W)$ related to $(t, s)$ by

8 This Calabi-Yau manifold served already as a prominent example in the understanding of typeIIA/heterotic duality [5] [31] and GEQFT's [32]. 
the mirror map. By calculating the period integrals and using the mirror map 9 we obtain the following result for the instanton expansion $\Delta_{n p}^{m(\psi)}=f_{s}$ that governs the non-perturbative gravitino masses:

$$
\begin{aligned}
\Delta_{n p}^{m(\psi)}= & e^{2 \pi i s}\left(2+2496 q_{t}^{1}+1941264 q_{t}^{2}+1327392512 q_{t}^{3}+861202986072 q_{t}^{4}+540194037151104 q_{t}^{5}+\ldots\right)+ \\
& e^{4 \pi i s}\left(\frac{1}{2}+448128 q_{t}^{2}+2654785024 q_{t}^{3}+5718020769540 q_{t}^{4}+8494210810708992 q_{t}^{5}+\ldots\right)+ \\
& e^{6 \pi i s}\left(\frac{2}{9}+\frac{347738368}{3} q_{t}^{3}+2583608958216 q_{t}^{4}+12741316216063488 q_{t}^{5}+\ldots\right)+ \\
& e^{8 \pi i s}\left(\frac{1}{8}+36401011968 q_{t}^{4}+2160776148604416 q_{t}^{5}+\ldots\right)+ \\
& \ldots
\end{aligned}
$$

It would be interesting to study the global non-perturbative vacuum structure using this exact information. In fact the coefficient functions $f_{k}\left(q_{t}\right)$ of the $k$-th instanton term are generally modular functions of the heterotic modular group, which is known for certain compactifications 10 . This information might be sufficient to determine global properties of the associated scalar potential. In the following we will take a different route and analyze the vacuum structure directly in an expansion around the point particle limit of the SYM theory.

\subsection{Generalizations}

It is easy to argue that the pattern of non-perturbative supersymmetry breaking described in the above $S U(2)$ case is generic for any gauge group $G$. First note that we used only the universal sector of the dilaton and the graviphoton in the gauging and the generalization to general $G$ is therefore trivial. Consider now the electric component $\tilde{X}^{\Sigma}$ of the field theory section (3.5). On general grounds it is clear that the entries 1 and $t_{i}$ will not receive $s$ dependent instanton corrections. So the instanton corrections that lift the $\mathcal{N}=1$ vacuum are entirely due to the modifications of the remaining period

$$
\tilde{X}^{2}=\frac{\partial \mathcal{F}}{\partial s}=-C_{i j} t_{i} t_{j}+\ldots
$$

This period describes tree-level gauge kinetic terms, and its $s$ corrections nonperturbative field theory corrections to it plus additional non-perturbative gravitational and string effects in the full theory. Thus on pure field theory grounds, the

9 We refer again to refs. [26] 27] for details and references on calculations of this type.

10 A study of the modular functions for special cases can be found in [33]. 
$\mathcal{N}=1$ vacuum will be lifted in any $\mathcal{N}=2$ QFT with non-perturbative corrections coupled to string theory 11 . The only case left over are conformal QFT's with the exact gauge coupling being equal to the tree level coupling. Still in this case we expect that, after adding the coupling to gravity and breaking of conformal invariance as a consequence, $s$ dependent instantons appear in the string/gravity sector 2 . It is interesting to observe that the lift of the $\mathcal{N}=1$ vacuum seems to be closely related to the breaking of conformal invariance.

\section{Supersymmetry breaking in the point particle limit}

In the following we want to pin down the structure of supersymmetry breaking as seen by the low energy observable gauge theory. The string theory embedding predicts a special form of the non-perturbative scalar potential of the low energy gauge theory with the supersymmetry breaking parameters linked in a useful way to the string geometric moduli.

\subsection{What can be expected from the field theory}

Let us first ask what might be expected from what is known about supersymmetry breaking in field theory. Obviously, since we consider spontaneous breaking in the $\mathcal{N}=2$ supergravity theory which encodes the exact non-perturbative $\mathcal{N}=2 \mathrm{SYM}$ theory, the supersymmetry breakdown should have a consistent formulation in terms of the latter. There are three known patterns of supersymmetry breaking consistent with the holomorphic structure of the $\mathcal{N}=2$ theory.

The first one is the addition of the $\mathcal{N}=1$ supersymmetric adjoint mass term discussed in the original work of Seiberg and Witten [3]. It was argued there that this term drives the theory to the point in the Coulomb moduli where the monopole gets massless. The combined superpotenial including the monopole hypermultiplet $(m, \tilde{m})$ is

$$
W=\sqrt{2} a_{D} m \tilde{m}+m_{a d j} u .
$$

The minimum of the scalar potential is at $a_{D}=0, m=\tilde{m}=-\frac{m_{\text {adj }}}{\sqrt{2}} \frac{d u}{d a_{D}}$, where the monopole becomes massless and condenses. The condensation of the magnetically

11 We emphasize once again that the no-go theorem applies to the string effective supergravity, not to a pure field theory decoupled from string theory.

12 It follows from the general arguments in section 2 that this is indeed the case. 
charged monopoles leads to a mass for the magnetic gauge field and confinement of the electric fields à la t'Hooft [2].

Another very elegant and interesting approach to supersymmetry breaking starting from the holomorphic properties of the $\mathcal{N}=2$ theory has been considered in refs. [34]. Compatibility with the analycity properties of the exact field theory can be implemented by the introduction of so-called spurion fields with vev's that trigger the breaking. It was already argued there that the structure of the potential obtained in this way is in qualitative agreement with what one expects from a supergravity theory.

Indeed, we will find that the supersymmetry breaking in string theory is morally a generalization of the two mechanisms 13 . In particular the supersymmetry breaking parameters will depend in a specific way on the geometry of the transverse dimensions.

\subsection{String effective action}

To study the string effective theory near the point particle limit, we consider an expansion in $\alpha^{\prime} \sim 1 / M_{\text {str }}^{2}$, keeping the exact quantities including the infinite instanton series at each order. This approach has been introduced in [32] to derive the nonperturbative field theory results from string theory. Specifically one considers the limit of small $\epsilon$ with

$$
\frac{\Lambda}{M_{s t r}}=\epsilon, \quad \Lambda \sim M_{s t r} e^{-8 \pi^{2} / b_{1} g^{2}}=\text { fixed }
$$

where $b_{1}$ is the one loop beta coefficient of the SYM theory. Note that the tree level coupling $g$ at the string scale - equal to the volume of the base $B$ - behaves as $\operatorname{Im} s \sim-b_{1} \ln \epsilon$. It was further argued in [32] that in this limit the string effective action is described by a supergravity period vector $\Pi$ with building blocks

$$
1, \epsilon a^{k}, \epsilon a_{D}^{k}, \epsilon^{2} u, s, \epsilon^{2} s u, \quad k=1, \ldots, r
$$

where the $a^{k}\left(a_{D}^{k}\right)$ are the vev's of the scalar fields in the electric (magnetic) vector multiplets of the SYM theory. A crucial fact for the following is the appearance of $u=\left\langle\operatorname{Tr} \phi^{2}\right\rangle$ at order $\epsilon^{2}$. The dependence of $\Pi$ on $u$ will be generate the $\mathcal{N}=1$ supersymmetric mass term for the adjoint scalar in (5.1).

13 There are extra terms that resemble the soft breaking terms of the $\mathcal{N}=1$ spurion approach 35. 
The structure of the string effective action around the field theory limit turns out to be quite intricate. We will banish details of the supergravity calculations in the appendix and content ourself with an outline of the qualitative structure in the following. We start with the following ansatz for the symplectic section $\Pi=\left(X^{\Sigma}, F_{\Sigma}\right)$ :

$$
X^{\Sigma}=\left(\begin{array}{c}
f_{\alpha}+\epsilon^{2} c_{\alpha} u \\
\epsilon c a^{k}
\end{array}\right), \quad F_{\Sigma}=\left(\begin{array}{c}
2 \eta_{\alpha} f_{\alpha}(s+\text { const. }) \\
\epsilon c a_{D}^{k}
\end{array}\right),
$$

where $\eta=-,+,+, \ldots$. The entries $X^{\alpha}, \alpha=1, \ldots, 2+m$ describe (together with the dual periods $F_{\alpha}$ ) the universal graviphoton/dilaton sector and $m$ extra scalar fields $t_{a}$ that parametrize the geometry of the transverse dimensions. On the other hand the entries $X^{k}, F_{k}, k=1, \ldots, r$ are associated to the $r=\operatorname{rk} G$ field theory periods. The expressions $f_{\alpha}, c_{\alpha}$ are so far arbitrary functions of the moduli $t_{a}$ but will have to satisfy some constraints imposed on the section $\Pi$ by $\mathcal{N}=2$ special geometry. The Kähler potential obtained from $\Pi$ has the form

$$
K=-\ln (\mathcal{V})+\frac{\epsilon^{2}}{\mathcal{V}} \mathcal{K}
$$

where the precise expression for $\mathcal{K}$ can be found in eq.(B.1) and the leading term is

$$
\begin{aligned}
\mathcal{V} & =V_{B} \cdot V_{T V}, \\
V_{B} & =i(s-\bar{s}+\text { const. }) \equiv \Sigma / 2, \\
V_{T V} & =2 \sum_{\alpha} \eta_{\alpha}\left|f_{\alpha}\right|^{2} \equiv 2 \Omega .
\end{aligned}
$$

In fact $\mathcal{V}$ is the volume of the Calabi-Yau manifold $M$. Note that the field theory dependent terms are contained in $\mathcal{K}$ and, apart from the inverse powers of $M_{s t r}$, suppressed by the overall inverse power of the volume $\mathcal{V}$. One can distinguish two different geometric scales contained in $\mathcal{V}$ which will be enter the supersymmetry breaking scales in the following. These are the volume of the base $V_{B} \sim \Sigma \sim \ln \epsilon$ on which the gauge fields propagate and the volume $V_{T V} \sim \Omega$ of the dimensions transverse to the field theory.

\subsection{A first look at the scalar potential}

The coupling of the hypermultiplets to the vector multiplets 4 induces a nontrivial scalar potential $V$ for the scalars [12]

$$
V=V_{\delta_{\Psi}}+V_{\delta_{\xi}}+V_{\delta_{\lambda}}=-3 P_{\Sigma}^{x} P_{\bar{\Lambda}}^{x} V^{\Sigma \bar{\Lambda}}+4 k_{\Sigma}^{u} h_{u v} k_{\bar{\Lambda}}^{v} V^{\Sigma \bar{\Lambda}}+P_{\Sigma}^{x} P_{\bar{\Lambda}}^{x} U^{\Sigma \bar{\Lambda}},
$$

14 See appendix A for more details. 
where the three terms are due to the gravitino, hyperino and gaugino variations, respectively. The couplings $P_{\Sigma}^{x}, x=1,2,3$ (and $k_{\Sigma}^{u}$ ) describe the interactions of the hypermultiplets $q^{u}$ with the four-dimensional vector multiplet $A_{\mu}^{\Sigma}$, where $\Sigma$ is a gauge index and $x$ an index of the $S U(2)_{R}$ symmetry. In the present context the couplings $P_{\alpha}^{x}$ in the universal dilaton/graviphoton sector parametrize the Super-Higgs effect discussed in the previous section. Since we will be interested in a region of the field theory moduli space where the monopoles are light, we must also add their couplings to the magnetic $U(1)$ factors of the field theory 15 . If $\left(m^{i}, \tilde{m}^{i}\right), i=1, \ldots, r$ denote the $r=\operatorname{rk} G$ monopole hypermultiplets with charges $q_{k}^{i}$, these couplings are described by $P_{k}^{x}=Q_{k}^{x}$ with

$$
\epsilon^{-2} Q_{k}^{x}=q_{k}^{i}\left(\bar{m}^{i}, \tilde{m}^{i}\right) \sigma^{x}\left(m^{i}, \overline{\tilde{m}}^{i}\right)^{T}
$$

The matrices $U$ and $V$ depend on the scalars $z_{i}$ in the vector multiplets. As their general form is quite involved we will restrict to give explicit expressions along the way when needed.

\section{The cosmological term}

The leading term in the scalar potential arises from the Super-Higgs effect in the universal sector and does not depend on the field theory moduli. It represents the cosmological constant from the view of the brane world. Its moduli dependence is described by the $\epsilon^{0}$-piece of the matrices $U$ and $V$ :

$$
e^{-K} V_{0}^{\alpha \bar{\beta}}=f_{\alpha} \bar{f}_{\beta} \quad e^{-K} U_{0}^{\alpha \bar{\beta}}=w_{\alpha, \rho} g^{\rho \bar{\rho}} \bar{w}_{\bar{\beta}, \bar{\rho}}
$$

where we used $t_{\rho}=\left\{t_{a}, s\right\}$ to denote the non field theory moduli and $w_{\alpha, \rho}=K_{\rho} f_{\alpha}+$ $f_{\alpha, \rho}$. To avoid a cosmological constant of the order of the string scale, the sum of the leading contributions from the gravitino, hyperino and gaugino variations in (5.7) should vanish. There are various possibilities to achieve such a cancellation at special values of the vector and/or hypermultiplets.

In the following we will mostly separate the question of why the cosmological constant is so small compared to $M_{\text {susy }}$ from the study of the pattern supersymmetry breaking on the brane as a function of the couplings $P_{\alpha}^{x}$ that determine the SuperHiggs effect. Note that providing a solution to the cosmological constant problem in the present context would be rather significant as even non-perturbative effects are

15 We will write all following equations in the appropriate local magnetic variables which are equal to $a_{D}^{k}=\partial / \partial a^{k} \mathcal{F}$ in the UV region. 
included. Although we will not solve the problem of the cosmological constant we will observe some interesting interrelations between the supersymmetry breaking on the brane and the cosmological term and keep an eye on possible mechanisms for a cancellation of the latter.

\section{The $\mathcal{N}=1$ preserving adjoint mass term}

Let us consider for a moment a naive cancellation of the individual contributions to the cosmological term by imposing

$$
P_{\alpha}^{x} \cdot w_{\alpha, \rho}=0, \quad P_{\alpha}^{x} \cdot f_{\alpha}=0
$$

Assuming that eq.(5.10) holds, one finds for the leading piece of the scalar potential in the field theory limit:

$$
\begin{aligned}
\epsilon^{-4} V= & \frac{1}{2} \sum_{x}\left(Q_{l}^{x}+m_{a d j}^{x} u_{l}\right) \tau^{l \bar{k}}\left(Q_{k}^{x}+\bar{m}_{a d j}^{x} \bar{u}_{\bar{k}}\right) \\
& +2 q_{l}^{i} q_{k}^{i} a^{l} a^{\bar{k}}\left(\left|m^{i}\right|^{2}+\left|\tilde{m}^{i}\right|^{2}\right),
\end{aligned}
$$

where

$$
m_{a d j}^{x}=M_{s t r} \cdot \frac{P_{\alpha}^{x} c_{\alpha}}{c} .
$$

Eq. (5.11) describes precisely the scalar potential of the $\mathcal{N}=2$ field theory with a mass term $\sim m_{a d j}$ for the adjoint scalars in the vector multiplets. As argued in ref. [3], the $\mathcal{N}=2$ theory is driven to the point $a^{l}=0, \forall l$ in the Coulomb moduli, where $r$

mutually local monopoles become massless and condense with a vev $\sim \sqrt{m_{\text {adj }} \Lambda}$ in a new $\mathcal{N}=1$ supersymmetric vacuum.

\section{Relations from special geometry and breaking to $\mathcal{N}=0$}

Imposing the condition (5.10) not only cancels the leading order contribution to the scalar potential but also a number of extra terms at $\mathcal{O}\left(\epsilon^{4}\right)$. As the expression in eq.(5.11) is the most general one compatible with $\mathcal{N}=1$ supersymmetry these terms must necessarily break $\mathcal{N}=1 \rightarrow \mathcal{N}=0$ in the SYM theory.

An generic solution to (5.10) is the limit of infinitely large extra dimensions, as will be discussed below. It might appear that partial supersymmetry breaking to $\mathcal{N}=1$ with the adjoint mass term (5.12) is possible for any such solution. However this is in fact not true. The reason is that the general ansatz (5.4) for the supergravity section $\Pi$ must be subject to further conditions in order that $\Pi$ is a valid symplectic section 
of $\mathcal{N}=2$ special geometry. In the appendix $\mathrm{B}$ we find that these relations have the important implication

$$
P_{\alpha}^{x} \cdot f_{\alpha}=0 \Rightarrow m_{a d j}^{x} \sim P_{\alpha}^{x} \cdot c_{\alpha}=0
$$

Thus cancellation of the $\mathcal{N}=1$ supersymmetry breaking terms at $\mathcal{O}\left(\epsilon^{4}\right)$ implies also the cancellation of the leading $\mathcal{N}=1$ adjoint mass term. Therefore, contrary to what might have been naively expected, there will be no excessively large separation of the two breaking scales to $\mathcal{N}=1$ and $\mathcal{N}=0$, respectively.

As we pointed out already, the no-go theorem in the string effective supergravity does not imply a no-go theorem for the pure field theory completely decoupled from string theory. This decoupling can be achieved by taking a strictly infinite string scale $M_{s t r}=\infty$ which, by eq.(5.2), amounts to a non-compact limit in the (tangential) direction of the base. In this case certain terms dressed by inverse powers of $\Sigma \sim \ln \Lambda / M_{\text {str }}$ drop from the effective field theory potential and eq.(5.10) can be replaced by a weaker condition that can be satisfied and connects to the $\mathcal{N}=1$ pure field theory vacuum discussed in [23]. However note that in the field theory coupled to string theory the phenomenologically acceptable regime is $\ln \Lambda / M_{s t r} \approx 20$ and supersymmetry is broken by the terms with extra powers of $\Sigma$ (and in fact we will argue that the phenomenologically interesting region is in the range of moderately small $\Sigma$ ). This leads again to a quite restricted hierarchy for the breaking scales to $\mathcal{N}=1$ and $\mathcal{N}=0$, respectively

In appendix B we collect a few additional relations derived from special geometry which contain some useful information. In particular they imply that the scale of the supersymmetry breaking is given by

$$
m_{Q} \sim b_{1} \cdot \frac{P_{\alpha} f_{\alpha}}{\Omega}
$$

which is also equal to the scale of the monopole condensate. The fact that $m_{Q}$ is proportional to the one-loop beta function coefficient fits well the discussion in section 4.3 where we observed that the supersymmetry breakdown is apparently linked to the violation of conformal invariance. Moreover eq.(5.14) displays the important fact that the supersymmetry breaking scale tends to be suppressed by the volume of the transverse dimensions. 
Non-compact transverse dimensions

The defining data of the string effective theory is is the period vector $\Pi$ of a K3 fibered Calabi-Yau manifold. A special property of the leading periods $f_{\alpha}$ in the universal sector is that they are free of any instanton corrections arising from a finite transverse volume $\Omega$. The reason is that these $s$-independent pieces are identical to the periods of a K3 manifold which is of the same type as the generic fiber in the fibration. Moreover on the K3 manifold there are no instanton corrections to the period integrals as a consequence of the $(4,4)$ supersymmetry of the CFT. Explicitly this means that the periods $f_{\alpha}$ are polynomial in the $t_{a}$ of degree two with the precise form dictated by the K3 intersection matrix. Note that there are instanton corrections with an action proportional to the Kähler volumes $\left\{t_{a}\right\}$ in the K3 fibration.

Obviously, a solution to both conditions in (5.10) can not exist for generic moduli. It would be interesting to classify possible solutions on sub-slices of the moduli of all K3's, which would be tantamount for finding solutions for a vacuum with vanishing leading cosmological constant. In the next section we will study in detail the dependence of the scalar potential on the universal volume modulus $t$ of the transverse dimensions. In this case it is easy to verify that the only solution to (5.10) is the limit of infinitely large transverse dimensions, $t=\infty$. For the reasons described above this does not lead to partial supersymmetry breaking, however. Note that noncompact transverse volume should not be confused with the pure field theory case, which requires infinitely large tangential dimensions $V_{B} \sim \ln \Lambda / M_{s t r} \rightarrow \infty$.

\section{Supersymmetry breaking on the brane}

After having outlined some general features we give now details of the supersymmetry breaking in the effective SYM theory on the brane as a function of the couplings

$P_{\alpha}^{x}$ parametrizing the Super-Higgs effect in the universal string sector 16 . For concreteness we will restrict the description of the deformations of the transverse geometry to the generic volume modulus $t$. Introducing extra moduli will not change the effective theory in the sector of the SYM theory; the way that these extra moduli enter is in the leading, cosmological term.

16 Note that the following expressions describe the supersymmetry breaking on the brane for arbitrary general couplings and thus the distinction of patterns with partial or complete classical Super-Higgs effect in the string sector arises only after making a specific choice for them. 
The universal sector of the graviphoton, the dilaton and the universal modulus $t$ is described by the following ansatz for the functions $f_{\alpha}$ :

$$
f_{0}=\frac{1}{2}\left(1+t^{2}\right), \quad f_{1}=\frac{1}{2}\left(1-t^{2}\right), \quad f_{2}=t .
$$

Moreover it follows from the relations of special geometry that the coefficients of the $u$ dependent terms in the symplectic section (5.4) are

$$
c_{0}=-b, \quad c_{1}=b, \quad c_{2}=0, \quad \bar{c}_{0}=-c_{0},
$$

where $b \sim b_{1}$ with a numerical coefficient specified in (B.5). With this information one can determine the matrices $U$ and $V$ and the scalar potential eq.(5.7). Since its general form is at first sight involved, we will perform the analysis in various steps.

\subsection{Leading terms in a large $\Sigma$ expansion}

Let us start with the leading terms of the field theory potential in an expansion in powers of

$$
\Sigma=-\frac{b_{1}}{\pi} \ln \Lambda / M_{s t r}
$$

The motivation for this expansion is that for a very large string scale, the terms with lower powers of $\Sigma$ are suppressed 17 . In particular the pure field theory potential will correspond to keeping only the leading terms in the limit $\Sigma=\infty$. However for the theory coupled to gravity, the logarithmic dependence on $M_{s t r}$ implies relative small values of $\Sigma$ bounded from above by the value $\sim 10 b_{1}$ for $M_{s t r} \sim M_{p l}$. In fact, as we will argue that the supersymmetry breaking scale is proportional to the string scale and moreover the latter can well be in the TeV range in this "world on a brane" scenario, the value of $\Sigma$ can be actually quite small for a moderate to low value of the string scale. In this case the terms with lower powers in $\Sigma$ are relevant. However we find it still useful to display the structure of the potential by organizing it in powers of $\Sigma$, keeping in mind that this will only give a good physical hierarchy for extremely large values of $M_{\text {str }}$.

From the general expressions in appendix $\mathrm{C}$ one obtains the following leading term of the scalar potential in the SYM theory:

17 In fact the relative contribution of these terms might be underestimated by the naive formula (6.3), since it does not take into account threshold effects which are known to be sizable in string theory compactifications with large compact dimensions. 


$$
\begin{aligned}
\epsilon^{-4} V= & \frac{1}{2}\left\{Q_{l}^{-}+M_{l}^{-}\right\} \tau^{l \bar{k}}\left\{Q_{k}^{+}+M_{k}^{+}\right\}+\frac{1}{2}\left\{Q_{l}^{3}+M_{l}^{3}\right\} \tau^{l \bar{k}}\left\{Q_{k}^{3}+M_{k}^{3}\right\} \\
& +2 q_{l}^{i} q_{k}^{i} a^{l} a^{\bar{k}}\left(\left|m^{i}\right|^{2}+\left|\tilde{m}^{i}\right|^{2}\right) \\
& -\alpha \frac{i b}{2 \Omega^{2}}\left(u_{s}-\bar{u}_{\bar{s}}\right)+\left(\frac{i}{2 \Omega} \beta u_{s}+\text { c.c. }\right) .
\end{aligned}
$$

Here the superscripts \pm refer to complex combinations $x_{1} \pm i x_{2}$ of the real fields. In particular $Q_{l}^{-}=2 m^{i} \tilde{m}^{i} q_{l}^{i}$ is the holomorphic bilinear in the monopole fields and $Q_{l}^{+}=\left(Q_{l}^{-}\right)^{*}$. The supersymmetry breaking terms in the potential can be divided into

i) F-type terms:

The vev of the holomorphic bilinear term in the monopole fields will be determined by the quantity $M_{l}^{-}$which can be split into its holomorphic and harmonic pieces in the SYM fields, respectively:

$$
M_{l}^{-}=m_{\mathcal{N}=1} u_{l}+m_{\mathcal{N}=0}\left(u_{l}-\text { c.c. }\right) .
$$

The holomorphic piece

$$
m_{\mathcal{N}=1}=\frac{b}{\bar{c} \Omega} P_{\alpha}^{-}\left(f_{\alpha}-\bar{f}_{\bar{\alpha}}\right)
$$

represents the $\mathcal{N}=1$ preserving adjoint mass term depending on the transverse volume as dictated by (6.1). On the other hand, the harmonic piece

$$
m_{\mathcal{N}=0}=\frac{b}{c \Omega} P_{\alpha}^{-} f_{\alpha}
$$

induces a soft breaking of supersymmetry.

ii) D-type terms:

The alignment of the vev's of the scalar components of the individual chiral $\mathcal{N}=1$ multiplets in the monopole hypermultiplet are determined by the remaining component $M_{l}^{3}=\zeta u_{l}+$ c.c. with

$$
\zeta=\frac{b}{c \Omega} P_{\alpha}^{3} \bar{f}_{\bar{\alpha}}
$$

iii) Remaining terms:

In addition there are further soft-breaking terms that depend on $u_{s}$ with coefficients

$$
\begin{array}{r}
\alpha=\frac{\Omega^{2}}{b^{2}}|c|^{2}\left(\frac{1}{2}\left|m_{\mathcal{N}=0}\right|^{2}+\frac{1}{2}\left|m_{\mathcal{N}=0}+m_{\mathcal{N}=1}\right|^{2}+|\zeta|^{2}\right), \\
\beta=\frac{\Omega}{b}\left(\frac{1}{2} c\left(m_{\mathcal{N}=1}+m_{\mathcal{N}=0}\right) C^{+}+\frac{1}{2} \bar{c} \bar{m}_{\mathcal{N}=0} C^{-}-c \zeta C^{3}\right),
\end{array}
$$


where $C^{x}=P_{\alpha}^{x} c_{\alpha}$. A nice heuristic interpretation of these terms can be given as follows. Let us consider the $\mathcal{N}=1$ supersymmetric term in (6.5). In the field theory this term can be associated with a superpotential term $u m_{\mathcal{N}=1}$ in eq.(5.1). At the point where the $\mathcal{N}=1$ vacuum branches off, $u=\omega_{G} \Lambda^{2}$, where $\omega_{G}$ is a $c_{2}(G)$-th root of unity. Moreover the scale of the $\mathcal{N}=1$ theory is related to that of the $\mathcal{N}=2$ theory by $\Lambda_{\mathcal{N}=1}^{3}=m_{\mathcal{N}=1} \Lambda^{2}$. Thus the superpotential term $u m_{\mathcal{N}=1}$, evaluated at the $\mathcal{N}=1$ point of the Coulomb branch is

$$
W=\omega_{G} \Lambda_{\mathcal{N}=1}^{3} .
$$

So remarkably enough, evaluating the superpotential of ref.[3] at the extremum with respect to the SYM fields, gives precisely the expected dynamical generated superpotential in the $\mathcal{N}=1$ theory. In the string theory context there are further contributions from the superpotential (6.10) to the scalar potential because $\Lambda \sim e^{i \gamma s}$ is to be treated as a field rather than a constant. These terms have the form of the $u_{s}$ dependent terms in the scalar potential (6.4).

To determine the scale of supersymmetry breaking, note that $\partial \tau^{l \bar{k}} / \partial a^{n}$ is very large in a region with light monopoles, $a^{k} \sim 0$. As a consequence, minimization with respect to the fields $a^{k}$ will require an adjustment of the monopole bilinears $Q_{l}^{x} \sim M_{l}^{x}$ to a high precision. Thus the generic scale of the monopole condensate and the supersymmetry breakdown is given by

$$
M_{\text {susy }} \sim \frac{b_{1}}{\Omega} P_{\alpha}^{x} f_{\alpha} M_{\text {str }} \sim \frac{b_{1}}{\Omega} M_{\text {str }},
$$

where in the second expression we have assumed that the couplings in the universal string sector are of order one in string units. As we will discuss in the next section, this is indeed the case if these couplings $P_{\alpha}^{x}$ arise from certain background fields in the string theory compactification.

The $\mathcal{N}=1$ pure field theory vacuum 1

If we are not interested in string theory but pure field theory, we can take the decoupling limit of infinite string mass which implies $\Sigma \rightarrow \infty$ by eq.(5.2). Only the leading terms (6.4) survive in this pure field theory case. This case was considered in [23] and it was asserted that a solution with $\mathcal{N}=1$ supersymmetry exists. Note that

18 I thank Cumrun Vafa for conversations on the following issues. 
there are many choices for the Super-Higgs effect that lead to partial supersymmetry breaking in the classical supergravity and break the remaining supersymmetry only by instanton effects. However only a very special subset will be related to a vacuum of the $\mathcal{N}=1$ pure field theory at $M_{\text {str }}=\infty$.

A choice of or RR-fluxes (or Super-Higgs effect) that cancels all supersymmetry breaking terms in the leading part of the potential must correspond to $\zeta=m_{\mathcal{N}=0}=0$ and values for $\alpha$ and $\beta$ such that the last terms in (6.4) cancel. From eqs.(6.7) - (6.9) we find that the solution is given by

$$
P_{0}^{-}=-\frac{1+t_{0}^{2}}{1-t_{0}^{2}} P_{1}^{-}, \quad P_{2}^{-}=\frac{2 t_{0}}{1-t_{0}^{2}} P_{1}^{-}, \quad P_{\alpha}^{3}=0, \forall \alpha
$$

The meaning of this equations is that for fixed $t_{0}$, the choice $(6.12)$ of the Super-Higgs cancels the supersymmetry breaking terms in the leading potential at $t=t_{0}$.

Let us summarize some relevant properties of the solution (6.12) that represents a special configuration of RR-fluxes that preserve $\mathcal{N}=1$ in the decoupled field theory at $M_{s t r}=\infty$. (1) It corresponds to a special fine tuning of fluxes dual to 0,2 and 4-form charges on the non-compact manifold. The leading contribution in the non-compact transverse limit relevant for geometric engineering of $\mathcal{N}=1$ QFT's is from the flux dual to the 0 and 4 -form charges (this outcome is slightly different from the 2-form flux proposed in [23]). (2) The solution exists already at finite $t$. The relevant decoupling is due to the infinite volume of the dimension parallel to the brane, $\operatorname{Im} s \sim \ln \Lambda / M_{s t r} \rightarrow \infty$ which gives an infinite mass to the string states. This is in nice agreement with our previous assertion that the breaking to $\mathcal{N}=0$ is indeed only due to the $s$-dependent instantons in the string sector, which are nonperturbative in the field theory coupling (rather than instantons from the, in string units, finite transverse volume). (3) For finite $M_{\text {str }}$ there are extra terms discussed in the next section that come with additional powers of $1 / \Sigma$ and break supersymmetry. Canceling the leading terms by (6.12) leads to an extra logarithmic dependence of the supersymmetry breaking scale on $M_{s t r}$. It is interesting to mention that the overall coefficient of these subleading terms depends on the hypermultiplet involved in the Super-Higgs effect (the value of $\lambda$ defined in the next paragraph). For the hypermultiplet involved in the string theory Super-Higgs effect this coefficient is nonzero [13] 36]. If the coefficient would have been zero, supersymmetry breaking would have been postponed to the next order of $\Lambda / M_{s t r}$. 


\subsection{Subleading terms and a vanishing leading cosmological constant}

Subleading terms in the $\Sigma$ expansion can be straightforwardly added using the expressions in appendix C. For the purpose of displaying their structure however it is useful to temporarily make a concrete and simple choice for the Super-Higgs effect. For a physically motivated illustration let us cancel the leading cosmological constant in the large volume limit by imposing eq. (5.10) on the leading terms. This is achieved by

$$
P_{0}^{1}=P_{1}^{1} \equiv m / M_{s t r}, \quad P_{\alpha}^{x}=0, \text { else, }
$$

were we have used a $S U(2)$ rotation to fix the direction of the triplet of Killing prepotentials. To treat the hyperino contribution in (5.7) on the same footing with the gaugino and gravitino we will use the relation $k_{\Sigma}^{u} h_{u v} k_{\Lambda}^{v}=\lambda P_{\Lambda}^{x} P_{\Sigma}^{x}$. This simplification is justified for the couplings in string theory with $\lambda=4$, as will be outlined in the next section. The full field theory dependent scalar potential for the Super-Higgs determined by 6.13 is

$$
\begin{aligned}
\epsilon^{-4} V= & \frac{1}{2}\left\{Q_{k}^{1}-\frac{\hat{M}}{|c|^{2}} u_{k}^{I}\right\} \tau^{k \bar{l}}\left\{Q_{l}^{1}-\frac{\hat{M}}{|c|^{2}} u_{l}^{R}\right\}+\frac{1}{2} \tau^{k \bar{l}}\left(Q_{k}^{2} Q_{l}^{2}+Q_{k}^{3} Q_{l}^{3}\right) \\
& +\left\{\gamma\left(2 u-a^{l} u_{l}\right) \frac{i \hat{M}^{2}}{2 b}+c . c .\right\}+2\left(a^{l} q_{l}^{i}\right)\left(\bar{a}^{\bar{k}} q_{k}^{i}\right)\left(\left|m^{i}\right|^{2}+\left|\tilde{m}^{i}\right|^{2}\right) \\
& -\left\{\frac{2 M^{2}}{b \Sigma}\left(1-\frac{2 i}{\gamma \Sigma}\right) u+\text { c.c. }\right\} \\
& -\left\{2 c \frac{M}{b \Sigma} Q_{l}^{1} a^{l}+\text { c.c. }\right\}+2 i|c|^{2} \frac{M^{2}}{b^{2} \Sigma^{2}}\left(a_{D}^{l} \bar{a}^{l}-\text { c.c. }\right)+\lambda \frac{M^{2}}{b^{2} \Sigma^{2}} \mathcal{K} .
\end{aligned}
$$

Here $u_{k}^{I}=c u_{k}+\bar{c} \bar{u}_{\bar{k}}$ and

$$
M=\frac{b}{\Omega} \frac{m}{\Lambda}, \quad \hat{M}=\left(1-\frac{4 i}{\gamma \Sigma}\right) M .
$$

Let us compare this result with the soft breaking terms discussed in the context of SYM theories in the literature. The first two lines of eq.(6.14) have the form of soft breaking terms generated by the spurion approach of [34]. The term in the third line is of the form generated by the $\mathcal{N}=1$ spurion of [35]. The coefficients of these breaking terms are related in the string effective theory which can be thought of explicitly determining the vev's of the spurion fields. The fourth line contains some additional soft breaking terms.

Note that the $\mathcal{N}=1$ adjoint mass term is absent only because of the choice of couplings (6.13) and as a consequence of eqs.(5.12) and (6.2). 


\subsection{Minima of the potential}

Ultimately, all moduli including the numerical values of $\Sigma$ and $\Omega$ should be fixed by the minima of the potential. The minimization with respect to the field theory moduli is relatively straightforward and reduces approximately to $Q_{l}^{x} \sim M_{l}^{x}$ with small corrections from the additional finite terms near the monopole point. In the special case with $m_{\mathcal{N}=1}=0$ above, the leading potential in a large $\Sigma$ expansion is of the form of the soft supersymmetry breaking considered in refs. [34], which include a detailed numeric study of the vacuum structure for the gauge group $S U(2)$. By comparison, the spurion vev should be identified with the scale $M_{\text {susy }}$ in (6.11).

To determine the string vacuum, one needs also to vary with respect to the dilaton $s$ and the transverse volume modulus $t$. This brings us back to the question of the cosmological term. We will not solve the question of minimization for these fields, which we leave for the future, but point out some qualitative features. As for the dilaton, a stabilization is possible due to the subleading terms in $\Sigma$ with explicit $s$ dependence. Note that there are also further calculable terms at order $\epsilon^{6}$ and higher which depend non-trivially on $s$. Thus although we do not know in the moment which value of $s$ corresponds to a minimum, there is no obstruction in principle to answer this question.

A similar situation holds for the volume modulus $t$ of the large extra dimensions. For a fixed $M_{\text {susy }} \sim 1 \mathrm{TeV}$, the behavior of the typical inverse radius vs. $M_{\text {str }}$ is plotted in the figure below.

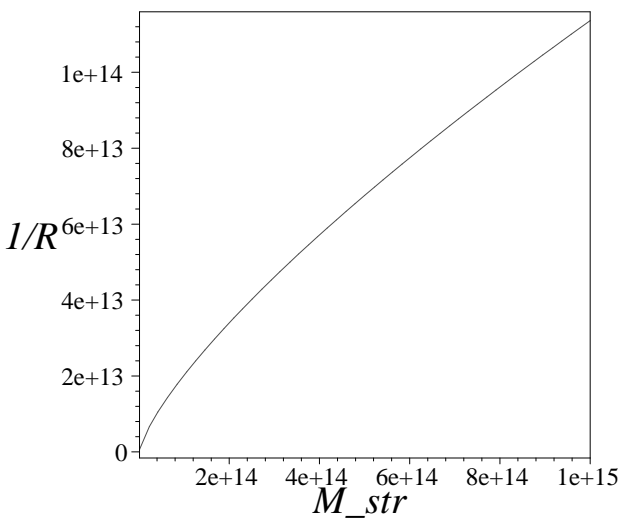


In particular, for small a $M_{s t r}$ in the TeV range, the average radius is close to $M_{s t r}^{-1}$ while it is larger by a factor of $\sim 10^{4}$ for a string scale equal to the Planck scale. Since the scale of supersymmetry breaking is $M_{s u s y} \sim V_{T V}^{-1}$ one suspects there is no local minimum for the volume modulus and thus $t$ will run away to infinity to restore supersymmetry. A natural idea to fix the minimization of the transverse volume is to link it to the minimization of the field theory moduli space [37]. In other words, we can use, as in the compactification discussed in section 4 , the fact that the volume modulus itself can correspond to one of the vev's of the field theory gauge group. Since the transverse volume is of order one in string units in such a model, one expects a relation

$$
M_{\text {susy }} \sim M_{\text {str }} \sim R^{-1} .
$$

Therefore this class of compactifications requires the string scale to be equal to the supersymmetry breaking scale in the $\mathrm{TeV}$ range and predicts it to be equal to the scale of the extra large dimensions.

\section{Geometric charges in string theory and why $M_{s t r}$ should be small}

In the previous sections we have described the supersymmetry breakdown as a consequence of a Super-Higgs effect in the universal sector, parametrized by the couplings $P_{\alpha}^{x}$. Let us finally comment on some important details of the relation of these couplings to the background fields in string theory.

In ref. [13] it was shown that backgrounds of RR $2 p$-forms in the type IIA theory induce charges of the dilaton hypermultiplet w.r.t. a gauge symmetry of the vector multiplets specified by the cohomology class of the background. From the equations in 113 it also follows that $\lambda=4$ for this case. Due to the special property of the RR sector in the type II theory these couplings are suppressed by a factor of the string coupling $\lambda_{I I}$.

This is an extremely important detail from the world on a brane point of view since $i$ ) the string scale $M_{\text {str }}$ may be in the $\mathrm{TeV}$ range in this scenario[38]; ii) the supersymmetry breaking scale (6.11) is of the order of $M_{s t r}$ rather than $M_{p l}$. In fact the relation between these two scales is given by dimensional reduction as

$$
M_{p l}^{2} \sim \frac{\Sigma \Omega}{\lambda_{I I}^{2}} M_{s t r}^{2}
$$


The special origin of the SYM theory - its origin in the RR sector which makes its coupling independent of $\lambda_{I I}$ and its localization on a hypersurface which makes its coupling independent of the transverse volume $\Omega$ - has the consequence that the values of $\Omega$ and $\lambda_{I I}$ remain largely undetermined by phenomenological requests. This allows for a large separation of the string scale and the Planck scale arising from a large transverse volume $\Omega$ and/or a small string coupling $\lambda_{I I}$.

After supersymmetry breaking, even a small mismatch in the cancellation of the cosmological constant in the field theory will drive the string scale to as small a value as possible. In other words not only is it consistent to have strings at the $\mathrm{TeV}$ scale in this world on a brane scenario, but, after the supersymmetry breakdown, a low string scale may well be energetically favored. In fact the solution to the cosmological constant problem should tell us why $M_{\text {str }} \sim$ a few TeV instead of being zero.

\section{Hidden sectors}

A type IIB version of the backgrounds of ref.[13], with a generalization to also include NS backgrounds has been given in refs. 36] 23] 19. In ref. 23] the coupling of the universal hypermultiplet to the vector gauge symmetries was derived from the BPS tension of NS and RR 5 branes in the presence of such backgrounds. As the tension of the NS brane lacks the suppression factor $\lambda_{I I}$, NS backgrounds will naturally lead to a supersymmetry breaking scale $\sim M_{p l}$. A low supersymmetry breaking scale could still be obtained by breaking first supersymmetry on a "hidden singularity" and then transmitting the breakdown by gravitational interactions to the observable branes, similarly as in refs. [1].

\section{Outlook}

Using the appropriate geometric singularities of ref.[17], there are no obvious obstacles to model a $\mathcal{N}=2$ supersymmetric string theory embedding of the gauge group of the

19 To compare the results of these two papers, which in fact seem to disagree in that the scalar potential of the [23] is $S L(2, \mathbf{Z})$ invariant whereas the one of [36] is not, one needs to rewrite the $\mathcal{N}=2$ scalar potential in terms of a $\mathcal{N}=1$ superpotential, a task that appears to have been settled only in very special cases, see [39]. We propose the general form of the $\mathcal{N}=1$ superpotential to be $W=P_{\Sigma} X^{\Sigma}-\tilde{P}^{\Sigma} F_{\Sigma}$, where $P_{\Sigma}=P_{\Sigma}^{1}+i P_{\Sigma}^{2}$ is the complex combination of the real Killing prepotentials and we have included a similar term $\tilde{P}^{\Sigma}$ for the couplings to the magnetic fields to ensure symplectic covariance. To verify the consistency of this ansatz, the use of the supersymmetric Ward-identity in eq.(2.97) of ref. 40 is essential. 
standard model on a brane and its matter spectrum. Breaking supersymmetry in the way described in this paper leads to a generalized, calculable scalar potential depending on the parameters of this "standard model". Apart from questions of details, e.g. how natural the standard model spectrum appears in the context of geometric singularities, higher derivative corrections etc, this appears to be an unexpectedly accomplishable scenario for the study of a phenomenologically relevant string vacuum with calculable, dynamically fixed mass and coupling parameters.

\section{Acknowledgments:}

I am grateful to Sheldon Katz, Wolfgang Lerche and Cumrun Vafa for essential comments and conversations. I would also like to thank Luis Álvarez-Gaumé, Costas Kounnas, Hans-Peter Nilles, Antoine van Proeyen and Stephan Stieberger for valuable discussions and comments.

\section{Appendix A. A brief review of the $\mathcal{N}=2$ scalar potential}

Apart from the vector multiplets, the $\mathcal{N}=2$ supergravity may contain a number $n_{H}$ of matter hypermultiplets $q^{u}$, with the $4 n_{H}$ scalars components parametrizing a quaternionic manifold $\mathcal{M}_{H}$. The $\mathcal{N}=2$ supergravity allows for couplings of the hypers to the vectors by gauging isometries of $\mathcal{M}_{H}$. In other words, hypermultiplets can be charged under the $U(1)^{n_{V}+1}$ gauge symmetry

$$
q^{u} \rightarrow q^{u}+k_{\Sigma}^{u} \epsilon^{\Sigma}
$$

with the Killing vector $k_{\Sigma}^{u}$ defining the charge of $q^{u}$ under the $\Sigma$-th $U(1)$ symmetry 20 . We refer to [41] for a most general and modern account of the combined $\mathcal{N}=2$ effective action and a detailed list of references.

The supersymmetry variations of the gauginos $\lambda_{A}^{\bar{a}}$, the hyperinos $\xi^{\alpha}$ and the gravitino $\psi_{A \mu}$ depend on the scalars $z_{i}$ as:

$$
\delta \lambda_{A}^{\bar{a}}=W_{A B}^{\bar{a}} \eta^{B}, \quad \delta \xi^{\alpha}=N_{A}^{\alpha} \eta^{A}, \quad \delta \psi_{A \mu}=i S_{A B} \gamma_{\mu} \eta^{B}
$$

20 In particular FI terms can be included in this description by gauging a "trivial" hypermultiplet. 
with

$$
\begin{aligned}
W_{A B}^{\bar{a}} & =i e^{K / 2}\left(\sigma^{x} \epsilon\right)_{A B} P_{\Sigma}^{x}\left(\partial_{b}+\partial_{b} K\right) X^{\Sigma} g^{b \bar{a}} \\
N_{A}^{\alpha} & =2 U_{u}^{\alpha B} \epsilon_{B A} e^{K / 2} k_{\Sigma}^{u} X^{\Sigma} \\
S_{A B} & =-\frac{1}{2}\left(\sigma^{x} \epsilon\right)_{A B} P_{\Sigma}^{x} e^{K / 2} X^{\Sigma} .
\end{aligned}
$$

Here $A=1,2, \Sigma=0, \ldots, n_{V}, a=1, \ldots, n_{V}, K$ is the Kähler potential, $U_{u}^{\alpha B}$ the symplectic vielbein and moreover the $P_{\Sigma}^{x}, x=1,2,3$ a triplet of Killing prepotentials associated to the gauging (A.1).

The coupling (A.1) of the hypermultiplets to the vector multiplets induces a nontrivial scalar potential $V$ for the scalars [12] which can be written in terms of the supersymmetry variations as

$$
\begin{aligned}
V= & -6 \operatorname{tr} S S^{*}+\operatorname{tr} N^{\dagger} N+\frac{1}{2} g_{a \bar{b}} \operatorname{tr}\left(W^{\bar{a}}\right)^{*} W^{\bar{a}}= \\
& \left(-3 P_{\Sigma}^{x} P_{\bar{\Lambda}}^{x}+4 k_{\Sigma}^{u} h_{u v} k_{\bar{\Lambda}}^{v}\right) V^{\Sigma \bar{\Lambda}}+P_{\Sigma}^{x} P_{\bar{\Lambda}}^{x} U^{\Sigma \bar{\Lambda}}
\end{aligned}
$$

with

$$
V^{\Sigma \bar{\Lambda}}=e^{K} X^{\Sigma} \bar{X}^{\bar{\Lambda}}, \quad U^{\Sigma \bar{\Lambda}}=f_{i}^{\Sigma} g^{i \bar{j}} \bar{f}_{\bar{j}}^{\bar{\Lambda}}, \quad f_{i}^{\Sigma}=e^{K / 2}\left(\partial_{i}+K_{i}\right) X^{\Sigma} .
$$

\section{Appendix B. Special geometry of the string effective GEQFT's}

To determine the effective string theory we need the precise form of the section $\Pi$. The large $s$ behavior (3.3) and the consistency of the supergravity monodromies with the monodromies of the embedded field theory imply the general form (5.4)

$$
X^{\Sigma}=\left(\begin{array}{c}
f_{\alpha}+\epsilon^{2} c_{\alpha} u \\
\epsilon c a^{k}
\end{array}\right), \quad F_{\Sigma}=\left(\begin{array}{c}
2 \eta_{\alpha} f_{\alpha}(s+\text { const. }) \\
\epsilon c a_{D}^{k}
\end{array}\right) .
$$

The Kähler potential has the $\epsilon$ expansion

$$
K=-\ln i\left(\bar{X}^{\Sigma} F_{\Sigma}-X^{\Sigma} \bar{F}_{\Sigma}\right)=-\ln (\mathcal{V})+\frac{\epsilon^{2}}{\mathcal{V}} \mathcal{K}
$$

with $\mathcal{V}$ given in (5.6), $A=\sum \eta_{\alpha} \bar{f}_{\alpha} c_{\alpha}$ and

$$
\mathcal{K}=i|c|^{2}\left(a^{k} \bar{a}_{D}^{k}-\bar{a}^{k} a_{D}^{k}\right)+2 i(A \bar{s} u-c . c .)+h(u, s)+\bar{h}(\bar{u}, \bar{s}) .
$$

The moduli dependent quantity $\Omega=\sum_{\alpha} \eta_{\alpha}\left|f_{\alpha}\right|^{2}$ describes the volume of the transverse dimensions. From the above expressions we can determine the matrices $U$ and $V$ entering the scalar potential (5.7). 


\section{Special geometry of $\Pi$}

Special geometry implies some important conditions on the functions appearing in the ansatz for $\Pi$ (5.4). From the supergravity identity

$$
U^{\Lambda \bar{\Sigma}}=-\frac{1}{2}(\operatorname{Im} \mathcal{N})^{\Lambda \Sigma}+V^{\Lambda \bar{\Sigma}},
$$

and the fact that $U$ is hermitian we see that $U-V$ must be a real symmetric matrix. Let us consider the leading $\epsilon^{1}$ term of $U^{\bar{\alpha} k}$. After some algebraic manipulations we obtain

$$
\begin{aligned}
& U_{1}^{\bar{\alpha} k}=V_{1}^{\bar{\alpha} k}+A^{\alpha} g^{\bar{k} l} u_{l}+B^{\alpha} g^{k \bar{l}} \bar{u}_{\bar{l}}, \\
& A^{\alpha}=-\frac{c b}{\mathcal{V} \Omega}\left\{\mathcal{V} \bar{f}_{\bar{\alpha}}+\frac{2 i}{\gamma} \bar{w}_{\bar{\alpha}, \bar{a}} g^{\bar{a} b} \Omega_{b}\right\}, \\
& B^{\alpha}=-\frac{c \bar{A}}{\mathcal{V} \Omega}\left\{\mathcal{V}\left(\bar{f}_{\bar{\alpha}}-\frac{\Omega}{\bar{A}} \bar{c}_{\bar{\alpha}}\right)-\bar{w}_{\bar{\alpha}, \bar{a}} g^{\bar{a} b}\left(2 i s \Omega\left(\frac{\bar{A}_{b}}{\bar{A}}-\frac{\Omega_{b}}{\Omega}\right)+\right.\right. \\
& \left.\quad \frac{\Omega}{\bar{A}}\left(\frac{\Omega_{b}}{\Omega} \bar{h}_{\bar{u}}-\bar{h}_{\bar{u} b}\right)\right\},
\end{aligned}
$$

where $w_{\alpha, a}=K_{a} f_{\alpha}+f_{\alpha, a}$. Moreover $b$ and $\gamma$ are two constants that are defined in the field theory:

$$
\begin{aligned}
\partial_{s} F_{F T} & =-\frac{2}{|c|^{2}} b u, \quad \Lambda=e^{\gamma s}, \\
\mathcal{F}_{F T} & =\frac{1}{2} \sum_{\mathrm{rk} G} a^{k} a_{D}^{k}+\frac{b}{|c|^{2} \gamma} u .
\end{aligned}
$$

In particular the constant $b$ is proportional to the one-loop beta function coefficient [42] [43].

From $\left(A^{\alpha}\right)^{*}=B^{\alpha}$ one can derive the following useful relations satisfied by the functions $f_{\alpha}, c_{\alpha}$ and $h$ appearing in the ansatz for $\Pi$ and in $\mathcal{K}$ :

$$
\begin{aligned}
h & =-2 i A u\left(s-\frac{1}{\gamma}\right)+\Omega u\left(\hat{c}_{1} s+\hat{c}_{2}\right), \quad \hat{c}_{i} \in \mathbf{C}, \\
0 & =\sum_{\alpha} f_{\alpha}^{2} \eta_{\alpha} \\
B & =\eta_{\alpha} f_{\alpha} c_{\alpha}=-\frac{c}{\bar{c}} b \\
\left|A^{2}\right| & =\left|B^{2}\right|
\end{aligned}
$$

and

$$
P_{\alpha} f_{\alpha}=0 \quad \Rightarrow \quad P_{\alpha} c_{\alpha}=0 .
$$




\section{Appendix C. The scalar potential for the universal volume modulus}

The $\epsilon$ expansion of the matrices $V$ and $\tilde{U}=U-V$ for the section $\Pi$ in (6.1) is given by

$$
\begin{aligned}
\underline{\mathcal{O}\left(\epsilon^{0}\right):} e^{-K} V_{0}^{\alpha \bar{\beta}}= & f_{\alpha} \bar{f}_{\beta} \quad e^{-K} U_{0}^{\alpha \bar{\beta}}=w_{\alpha, \rho} g^{\rho \bar{\rho}} \bar{w}_{\bar{\beta}, \bar{\rho}}, \quad e^{-K} U_{0}^{l \bar{k}}=\frac{\mathcal{V}}{2} \tau^{l \bar{k}} \\
\underline{\mathcal{O}\left(\epsilon^{1}\right):} e^{-K} V_{1}^{\alpha \bar{k}}= & f_{\alpha} \bar{c} \bar{a} \bar{k} \\
e^{-K} \tilde{U}_{1}^{\alpha \bar{k}}= & \frac{V}{2|c|^{2}} \tau^{\bar{k} l}\left\{A^{\alpha} u_{l}+c . c .\right\} \\
\underline{\mathcal{O}\left(\epsilon^{2}\right):} e^{-K} V_{2}^{\alpha \bar{\beta}}= & c_{\alpha} \bar{f}_{\beta} u+\bar{c}_{\beta} f_{\alpha} \bar{u}, \quad e^{-K} V_{2}^{k \bar{k}}=|c|^{2} a^{k} \bar{a}^{\bar{k}}, \\
e^{-K} \tilde{U}_{2}^{\alpha \bar{\beta}}= & \frac{V}{2|c|^{2}} X_{k}^{\alpha} \tau^{k \bar{l}} \bar{X}_{\bar{l}}^{\bar{\beta}}+\left\{\frac{i b \Sigma}{2}\left(\eta_{\alpha}-\frac{f_{\alpha}}{\Omega}\right) \bar{f}_{\bar{\beta}} u_{s}+h . c .\right\}+ \\
& \left\{-\frac{1}{\sqrt{2 \Omega}}\left(\mathcal{K}_{\bar{s}}+\frac{2 i}{\Sigma} \mathcal{K}\right) f_{\alpha} \bar{w}_{\bar{\beta}, \bar{t}}+h . c .\right\}+ \\
& \left\{i \sqrt{2 \Omega}\left(\eta_{\alpha} b u+\frac{\mathcal{K}}{\Omega \Sigma} f_{\alpha}\right) \bar{w}_{\bar{\beta}, \bar{t}}+h . c .\right\}-\frac{3 \mathcal{K}}{\Sigma} w_{\alpha, t} \bar{w}_{\bar{\beta}, \bar{t}},
\end{aligned}
$$

where $t_{\rho}=\{t, s\}$ and

$$
\begin{aligned}
A^{\alpha} & =-\frac{c b}{\Omega}\left\{\bar{f}_{\bar{\alpha}}+\frac{2 \sqrt{2 \Omega}}{\gamma \Sigma} \bar{w}_{\bar{\alpha}, \bar{t}}\right\}, \\
X_{k}^{\alpha} & =\frac{1}{\bar{c}}\left(A^{\alpha} u_{k}+\text { c.c. }\right)+\frac{2 \sqrt{2} i|c|^{2}}{\Sigma \sqrt{\Omega}} w_{\alpha, t} \tau_{k \bar{l}} \bar{a}^{\bar{l}}, \\
\tau_{k, \bar{l}} & =\operatorname{Im} a_{D, l}^{k} .
\end{aligned}
$$




\section{References}

[1] H.P. Nilles, Phys. Lett. $\underline{B 115}$ (1982) 193, Nucl. Phys. $\underline{B 217}$ (1983) 366;

S. Ferrara, L. Girardello and H.P. Nilles, Phys. Lett. $\underline{B 125}$ (1983) 457;

for a review and further references see H.P. Nilles, Int. J. Mod. Phys. $\underline{A 5}$ (1990) 4199.

[2] G. t'Hooft, Nucl. Phys. $\underline{B 190}$ (1981) 455.

[3] N. Seiberg and E. Witten, Nucl. Phys. $\underline{B 426}$ (1994) 19, erratum: ibid 430 (1994) 396.

[4] K. Konishi, Phys. Lett. B392 (1997) 101.

[5] S. Kachru and C. Vafa, Nucl. Phys. $\underline{B 450}$ (1995) 69.

[6] S. Ferrara, J. A. Harvey, A. Strominger and C. Vafa, Phys. Lett. B361 (1995) 59.

[7] S. Kachru and E. Silverstein, Nucl. Phys. B463 (1996) 369.

[8] C. Vafa and E. Witten, Nucl. Phys. Proc. Suppl. $\underline{46}$ (1996) 225.

[9] E. Kiritsis and C. Kounnas, Nucl. Phys. B503 (1997), 117;

E. Kiritsis, C. Kounnas, P. M. Petropoulos and J. Rizos, Nucl. Phys. $\underline{B 540}$ (1999) 87.

[10] S. Cecotti, L. Girardello and M. Porrati, Phys. Lett. $\underline{B 145}$ (1984) 61.

[11] S. Ferrara, L. Girardello and M. Porrati, Phys. Lett. $\underline{B 366}$ (1996) 155; Phys. Lett. $\underline{B 376}$ (1996) 275.

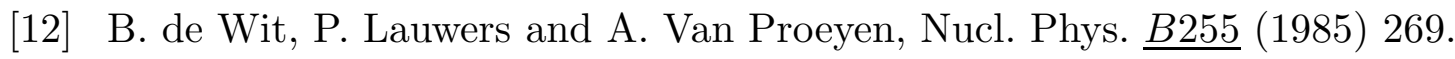

[13] J. Polchinski and A. Strominger, Phys. Lett. $\underline{B 388}$ (1996) 736.

[14] C. Bachas, A Way to break supersymmetry, hep-th/9503030;

I. Antoniadis, E. Gava, K.S. Narain and T.R. Taylor, Nucl. Phys. B511 (1998) 611.

[15] A. Klemm, W. Lerche, P. Mayr, C. Vafa, N. Warner, Nucl. Phys. $\underline{B 477}$ (1996) 746.

[16] S. Katz, A. Klemm and C. Vafa, Nucl. Phys. $\underline{B 497}$ (1997) 173.

[17] S. Katz, P. Mayr and C. Vafa, Adv. Theor. Math. Phys. 1 (1998) 53.

[18] E. Witten, Nucl. Phys. B500 (1997) 3.

[19] N. Arkani-Hamed, S. Dimopoulos and G. Dvali, Phys. Lett. $\underline{B 429}$ (1998) 263;

I. Antoniadis, N. Arkani-Hamed, S. Dimopoulos and G. Dvali, Phys. Lett. $\underline{B 436}$ (1998) 257. 
[20] S. Katz and C. Vafa, Nucl. Phys. B497 (1997) 196;

M. Bershadsky, A. Johansen, T. Pantev, V. Sadov and C. Vafa, Nucl. Phys. $\underline{\text { B505 }}$ (1997) 153;

P. Mayr and P. Berglund, Adv. Theor. Math. Phys. 2 (1999) 1307.

[21] J.L.F. Barbón, Phys. Lett. $\underline{B 402}$ (1997) 59.

[22] H.P. Nilles, Phys. Lett. $\underline{B 112}$ (1982) 455;

G. Veneziano and S. Yankielowicz, Phys. Lett. $\underline{B 113}$ (1982) 231.

[23] T.R. Taylor and C. Vafa, RR flux on Calabi-Yau and partial supersymmetry breaking, hep-th/9912152.

[24] A. Ceresole, R. D'Auria, S. Ferrara and A. Van Proeyen, Nucl. Phys. B4444 (1995) 92.

[25] I. Antoniadis, H. Partouche and T. R. Taylor, Phys. Lett. B372 (1996) 83.

[26] P. Candelas, X.C. De La Ossa, P.S. Green and L. Parkes, Nucl. Phys. B359 (1991) 21.

[27] Essays on Mirror Manifolds, (ed. S.T. Yau), Int. Press, Hong Kong, 1992.

[28] K. Hori and C. Vafa, Mirror symmetry, hep-th/0002222.

[29] R.L. Bryant and P.A. Griffiths, Some observations on the infinitesimal period relations for regular threefolds with trivial canonical bundle, in Arithmetic and Geometry Volume II (M. Artin and J. Tate, eds.), Progress in Math. 39, Birkhäuser, Boston-Basel-Berlin, 1983.

[30] P. Mayr, Fortsch. Phys. 47 (1998) 39.

[31] A. Klemm, W. Lerche and P. Mayr, Phys. Lett. B357 (1995) 313.

[32] S. Kachru, A. Klemm, W. Lerche, P. Mayr and C. Vafa, Nucl. Phys. B459 (1996) 537.

[33] B.H. Lian and S.-T. Yau, Nucl. Phys. Proc. Suppl. $\underline{46}$ (1996) 248;

M. Henningson and G. Moore, Nucl. Phys. $\underline{B 482}$ (1996) 187.

[34] L. Álvarez-Gaumé, J. Distler, C. Kounnas and M. Mariño, Int. J. Mod. Phys. $\underline{A 11}$ (1996) 4745;

L. Álvarez-Gaumé and M. Mariño, Int. J. Mod. Phys. $\underline{A 12}$ (1997) 975;

L. Álvarez-Gaumé, M. Mariño and F. Zamora, Int. J. Mod. Phys. A13 (1998) 403;

Int. J. Mod. Phys. $\underline{A 13}$ (1998) 1847.

[35] N. Evans, S.D.H. Hsu and M. Schwetz, Phys. Lett. B355 (1995) 475;

N. Evans, S.D.H. Hsu, M. Schwetz and S.B. Selipsky,Nucl. Phys. B456 (1995) 205

[36] J. Michelson, Nucl. Phys. B495 (1997) 127. 
[37] Work in progress.

[38] I. Antoniadis and B. Pioline, Nucl. Phys. B550 (1999) 41.

[39] J.P. Derendinger, S. Ferrara, A. Masiero and A. Van Proeyen, Phys. Lett. $\underline{B 140}$ (1984) 307;

E. Cremmer, C. Kounnas, A. Van Proeyen, J.P. Derendinger, S. Ferrara, B. de Wit and L. Girardello, Nucl. Phys. $\underline{B 250}$ (1985) 385.

[40] R. D'Auria, S. Ferrara and P. Fre, Nucl. Phys. B359 (1991) 705.

[41] L. Andrianopoli, M. Bertolini, A. Ceresole, R. D'Auria, S. Ferrara and P. Fré, J. Geom Phys. $\underline{23}$ (1997) 111; Nucl. Phys. $\underline{B 476}$ (1996) 397.

[42] M. Matone, Phys. Lett. $\underline{B 357}$ (1995) 342.

[43] J. Sonnenschein, S. Theisen and S. Yankielowicz, Phys. Lett. B367 (1996) 145. 